\title{
Article
}

\section{HER2-Positive Breast Cancer Patients with Pre-Treatment Axillary Involvement or Postmenopausal Status Benefit from Neoadjuvant Rather than Adjuvant Chemotherapy Plus Trastuzumab Regimens}

\author{
Enora Laas ${ }^{1}$, Arnaud Bresset ${ }^{2}{ }^{\mathbb{D}}$, Jean-Guillaume Féron ${ }^{1}$, Claire Le Gal ${ }^{1}$, Lauren Darrigues ${ }^{3}$, Florence Coussy ${ }^{4}$, \\ Beatriz Grandal ${ }^{3}$, Lucie Laot ${ }^{1}$, Jean-Yves Pierga ${ }^{4}$, Fabien Reyal ${ }^{1,3, *}$ and Anne-Sophie Hamy ${ }^{3,4}$ (D) \\ 1 Department of Surgery, Institut Curie, 5 rue d’Ulm, 75005 Paris, France; enora.laas@curie.fr (E.L.); \\ jeanguillaume.feron@curie.fr (J.-G.F.); claire.legal@curie.fr (C.L.G.); lucie.laot@aphp.fr (L.L.) \\ 2 Gynecology Department, Beaujon Hospital, 92210 Clichy, France; arnaud.bresset@aphp.fr \\ 3 Residual Tumor \& Response to Treatment Laboratory, RT2Lab, Translational Research Department, INSERM, \\ U932 Immunity and Cancer, Institut Curie, 75005 Paris, France; lauren.darrigues@curie.fr (L.D.); \\ beatriz.grandalrejo@curie.fr (B.G.); anne-sophie.hamy-petit@curie.fr (A.-S.H.) \\ 4 Department of Medical Oncology, Institut Curie, 75005 Paris, France; florence.coussy@curie.fr (F.C.); \\ jean-yves.pierga@curie.fr (J.-Y.P.) \\ * Correspondence: fabien.reyal@curie.fr; Tel.: +33-(0)-6-15-27-19-80
}

\section{check for} updates

Citation: Laas, E.; Bresset, A.; Féron, J.-G.; Le Gal, C.; Darrigues, L.; Coussy, F.; Grandal, B.; Laot, L.; Pierga, J.-Y.; Reyal, F.; et al. HER2-Positive Breast Cancer Patients with Pre-Treatment Axillary Involvement or Postmenopausal Status Benefit from Neoadjuvant Rather than Adjuvant Chemotherapy Plus Trastuzumab Regimens. Cancers 2021, 13, 370. https://doi.org/10.3390/ cancers 13030370

Received: 8 December 2020

Accepted: 16 January 2021

Published: 20 January 202

Publisher's Note: MDPI stays neutral with regard to jurisdictional claims in published maps and institutional affiliations.

Copyright: (c) 2021 by the authors. Licensee MDPI, Basel, Switzerland. This article is an open access article distributed under the terms and conditions of the Creative Commons Attribution (CC BY) license (https:// creativecommons.org/licenses/by/ $4.0 /)$.
Simple Summary: Neoadjuvant chemotherapy strategy (NAC) is a standard of care for Human Epidermal Growth Factor Receptor-2 (HER2)-positive early breast cancer but there is no proven beneficial evidence in terms of survival compared to the adjuvant chemotherapy strategy. Our retrospective study found a survival benefit in NAC strategy particularly in clinical Nodepositive and postmenopausal patients.

Abstract: Background: No survival benefit has yet been demonstrated for neoadjuvant chemotherapy (NAC) against HER2-positive tumors in patients with early breast cancer (BC). The objective of this study was to compare the prognosis of HER2-positive BC patients treated with NAC to that of patients treated with adjuvant chemotherapy (AC). Materials and methods: We retrospectively analyzed disease-free (DFS) and overall survival (OS) in 202 HER2-positive patients treated with NAC and 701 patients treated with AC. All patients received trastuzumab in addition to chemotherapy. Patient data were weighted by a propensity score to overcome selection bias. Results: After inverse probability of treatment weights (IPTW) adjustment, no difference in DFS ( $p=0.3$ ) was found between treatments for the total population. However, after multivariate analysis, an interaction was found between cN status and chemotherapy strategy (IPTW-corrected corrected Hazard ratio cHR $=0.52$, $\left.95 \% \mathrm{CI}(0.3-0.9), p_{\text {interaction }}=0.08\right)$ and between menopausal status and chemotherapy $(\mathrm{CT})$ strategy $\left.(\mathrm{cHR}=0.35,95 \% \mathrm{CI}(0.18-0.7)) p_{\text {interaction }}<0.01\right)$. NAC was more beneficial than AC strategy in $\mathrm{cN}$ positive patients and in postmenopausal patients. Moreover, after IPTW adjustment, the multivariate analysis showed that the neoadjuvant strategy conferred a significant OS benefit ( $\mathrm{cHR}=0.09,95 \% \mathrm{CI}$ [0.02-0.35], $p<0.001)$. Conclusion: In patients with HER2-positive BC, the NAC strategy is more beneficial than the AC strategy, particularly in $\mathrm{cN}$-positive and postmenopausal patients. NAC should be used as a first-line treatment for HER2-positive tumors.

Keywords: breast cancer; neoadjuvant chemotherapy; adjuvant chemotherapy; HER2-positive tumors

\section{Introduction}

Epidermal Growth Factor Receptor-2 (HER2)-positive breast cancers (BCs) are carcinomas characterized by the amplification and overexpression of the HER2 tyrosine kinase 
receptor gene (17q12), and they account for $15-20 \%$ of all BCs [1]. Before the advent of trastuzumab and anti-HER2-targeted therapies (i.e., lapatinib, pertuzumab and TDM-1), the rates of early distant metastatic events in these patients were high. Since the introduction of trastuzumab into routine use in the adjuvant setting in the middle of the first decade of this century, the combination of trastuzumab and chemotherapy has considerably improved the prognosis of patients with HER2-positive early BC [2].

Neoadjuvant chemotherapy (NAC) is now widely used in patients with early BC, to achieve breast-conserving surgery (BCS) through prior tumor size reduction, and it is now a standard of care for patients with HER2-positive BC [3-7]. In addition to BCS, NAC provides the possibility of in-vivo chemosensitivity tests of tumor burden and an opportunity to observe clinical and pathological responses in each patient. NAC is therefore an effective and informative strategy for testing novel therapies, providing an opportunity to adapt adjuvant strategy (TDM-1) [8,9].

No difference in overall survival (OS) has been detected to date between neoadjuvant and adjuvant strategies, neither in the total population of $\mathrm{BC}$ patients nor in the population of patients with HER2-positive tumors. Indeed, in pivotal trials addressing the equivalence between these two strategies, HER2 status was not routinely evaluated [10-12]. In an Early Breast Cancer Trialists' Collaborative Group EBCTCG meta-analysis pooling individual data from 4756 patients included in randomized trials of NAC vs. AC strategies $(n=10)$, no significant difference between these strategies was found for distant recurrence at 15 years (rate ratio 1.02 (95\% CI 0.92-1.14); $p=0.66)$, BC mortality (1.06 (0.95-1.18); $p=0.31$ ), or death from any cause (1.04 (0.94-1.15); $p=0.45)$, regardless of histological subtype [13].

The aim of this study was to compare the impact of NAC and AC strategies (in addition to trastuzumab in both groups) on survival outcomes, in HER2-positive BC patients, using a propensity score to avoid selection bias.

\section{Materials and Methods}

\subsection{Patient Selection}

Patients with T1-3N0-3M0 invasive HER2-positive tumors undergoing primary treatment with a combination of NAC and trastuzumab at Institut Curie between 1 January 2005 and 31 December $2012(n=202)$ (NEOREP cohort CNIL declaration number 1547270) were selected to constitute the NAC group. These patients were compared with 701 patients with T1-3N0-3M0 invasive HER2-positive breast tumors treated with a combination of AC and HER2-targeted therapies between 1 January 2005 and 31 December 2012, identified from the Institut Curie prospective breast cancer database. Unifocal, unilateral, non-recurrent, non-metastatic tumors were included. This study was approved by the Breast Cancer Study Group of Institut Curie and was conducted according to institutional and ethical rules concerning research on tissue specimens and patients. Informed consent from patients was not required. Information concerning clinical (age, menopausal status, body mass index, clinical tumor size, clinical nodal status) and tumor characteristics (histological size in the AC group and histological response to NAC, lymph node involvement, mitotic index, ki67, histological tumor grade, estrogen receptor (ER) status, progesterone receptor (PR) status, HER2 status) was retrieved from patients' medical records. Clinical size and clinical nodal status were used for the analysis, as they play a crucial role in decisions regarding tumor management.

\subsection{Tumor Samples}

Histological grade was determined according to the Elston-Ellis (EE) modification of the Scarff-Bloom-Richardson grading system [14] on post-operative tumor samples for the AC group and needle core biopsy specimens for the NAC group. Hormone receptor (HR) expression was analyzed by immunohistochemistry. Tumors were considered positive for ER or PR if $10 \%$ of the carcinoma cells displayed positive staining, as recommended by French guidelines [15]. Tumors were considered to be hormone receptor (HR)-positive if they were positive for either ER or PR, and HR-negative if they were negative for both ER 
and PR. HER2 status was determined according to American Society of Clinical Oncology (ASCO) recommendations [16]. Scores of $3+$ were considered positive, and scores of $1+/ 0$ were considered negative. Tumors with scores of $2+$ were further tested by Fluorescence In Situ Hybridization (FISH). We assessed HER2 gene amplification by calculating the mean HER2 signal per nucleus for a mean of 40 tumor cells per sample. A HER2/CEN17 ratio $\geq 2$ was considered positive and a ratio $<2$ negative.

\subsection{Treatment Protocol}

Patients were treated according to French national guidelines. The decision to administer NAC was made at a multidisciplinary medical meeting, in cases for which conservative surgery was not possible or for patients with high-risk factors (positive nodal status, high EE grade, high mitotic index, etc.). Chemotherapy regimens changed over time. In the adjuvant setting, chemotherapy was initiated four to eight weeks after surgery. The chemotherapy regimen was anthracycline-based in 101 patients (14\%), taxane-based in $12(2 \%)$, and a sequential anthracycline-taxane regimen (three cycles of epirubicin-cyclophosphamide followed by three cycles of docetaxel, or 12 cycles of paclitaxel weekly) was administered in $546(78 \%)$ patients. Trastuzumab was administered at a dose of $8 \mathrm{mg} / \mathrm{kg}$ (loading dose) and then $6 \mathrm{mg} / \mathrm{kg}$ every 3 weeks. Weekly trastuzumab treatment was then continued for one year.

In the neoadjuvant setting, three patients $(1 \%)$ had an anthracycline-based regimen, $16(8 \%)$ had a taxane-based regimen, and $175(87 \%)$ had a sequential anthracycline-taxane regimen (four cycles of epirubicin-cyclophosphamide followed by four cycles of docetaxel or 12 cycles of paclitaxel weekly). Trastuzumab was administered at a dose of $8 \mathrm{mg} / \mathrm{kg}$ (loading dose) and then $6 \mathrm{mg} / \mathrm{kg}$ every 3 weeks.

None of the patients received pertuzumab, since it was off-label in France during the period of the study, in the non-metastatic setting.

Surgery was performed three to six weeks after the last chemotherapy session. Weekly trastuzumab was then continued for one year after surgery.

In the adjuvant setting, sentinel lymph node biopsy (SLND) was performed in case of cN0 and axillary lymph node dissection (ALND) for patients who were cN-positive. In the neoadjuvant setting, during the study period, French and European recommendations stated for systematic ALND without SLND after NAC, so most patients underwent ALND [17,18].

Pathological complete response (pCR) was defined as no residual invasive/noninvasive cancer in the breast and nodes (ypT0-ypN0).

Patients received adjuvant radiotherapy according to national guidelines. The indications for radiotherapy were breast-conserving surgery, radical mastectomy in cases of initial T3 tumors, patients with axillary lymph node involvement, and patients with high-risk node-negative breast cancer. Adjuvant endocrine therapy (tamoxifen, aromatase inhibitor, or Gonadotropin-Releasing Hormone agonists) was prescribed when indicated to patients displaying hormonal receptor expression after radiotherapy. After completing treatment, patients were followed every four months for the first two years, every six months for the next three years, and then annually after five years. Follow-up consisted of a clinical examination associated with mammography and mammary ultrasound once per year.

\subsection{Study Endpoint}

The aim of the study was to analyze and compare prognosis and oncological outcomes between NAC and AC strategies. Disease-free survival (DFS) was defined as the time from surgery to locoregional, distant recurrence or death, whichever occurred first. Overall survival (OS) was defined as the time from surgery to death. Patients for whom none of these events were recorded were censored at the date of last known contact.

Distant recurrence-free survival (DRFS) was defined as the time from surgery to distant recurrence or death, whichever occurred first. 


\subsection{Statistical Analysis}

Qualitative variables were compared with Chi-squared or Fisher's exact tests, and quantitative variables were compared with Student's $t$-tests. A significance threshold of $5 \%$ was applied. As patients were not randomly allocated to the AC or NAC arms, a propensity score (PS) [19-22] was calculated to control for selection bias. The characteristics of the patients in the two groups at baseline were compared in univariate analysis. A multivariate logistic regression model was then used to generate the PS. The covariates included in the model were patient clinical characteristics (age, menopausal status, body mass index, initial clinical tumor size), histological characteristics at diagnosis (ElstonEllis grade), and initial clinical nodal status. We created balanced cohorts, using the inverse probability of treatment weights (IPTW), defined as the inverse of the propensity score for patients in the NAC population and $1 /(1$ - propensity score $)$ for patients in the AC population. The IPTW adjustment method is a valuable method to obtain unbiased estimates of average treatment effects $[23,24]$ and has already been used in breast cancer studies $[25,26]$. Weight outliers lying above the 99 th percentile were truncated to prevent sparse data. Missing data were handled by multiple imputation with chained equations. The benefit of NAC was estimated in the initial and IPTW-corrected populations, from Kaplan-Meier curves. Survival was compared between groups by performing log-rank tests on the initial population and a weighted univariate Cox model in the IPTW-corrected population [27]. An IPTW-corrected multivariate Cox model analysis was then performed, to take into account potential confounding factors not included in the PS (mostly surgical management). Hazard ratios for relapse, corrected by the IPTW procedure, are referred to as corrected HR (cHR) hereafter. We tested the hypothesis of potentially different effects of NAC in different subgroups, by including pairwise interaction terms in the Cox model. Due to the lack of statistical power for analyzing interactions [28], a $p$-value of 0.10 or lower was considered statistically significant. Analyses were performed with R software, version 3.3 [29].

\section{Results}

Overall, 903 patients with HER2-positive BC underwent NAC $(n=202)$ or AC $(n=701)$ with HER2-trastuzumab (Table 1). Before IPTW correction, patients from the NAC group were younger than those from the AC group (48.2 vs. 53.6 years old; $p<0.0001$ ), had tumors of a larger median size ( $40 \mathrm{vs.} 20 \mathrm{~mm} ; p<0.001)$, were more likely to have clinical nodal involvement $(60.1 \%$ vs. $18.3 \%, p<0.0001)$, and to be treated by BCS $(70.4 \%$ vs. $40.9 \%$; $p<0.0001$ ). Hormone receptor status did not differ between groups ( $55 \%$ of patients in each group had HR-positive tumors, $p=0.9$ ), and was not balanced between pre- and post-menopausal patients $(60 \% \mathrm{HR}$-positive for premenopausal patients vs. $50 \%$ for postmenopausal patients, $p=0.03$, Figure 1). The pathological complete response (pCR) rate was $38.5 \%$ in the NAC group.

The median time from $B C$ diagnosis to treatment was significantly lower in the NAC group than in the $\mathrm{AC}$ group (time from $\mathrm{BC}$ diagnosis to first chemotherapy: 21 days vs. 83 days, $p<0.001$, Figure A1A; time from BC diagnosis and first trastuzumab injection: 103 days vs. 156 days, Figure A1B). With a median follow-up of 39.2 months for the NAC group and 48.7 months for the AC group, there were 74 events (NAC: $n=20 ; \mathrm{AC}: n=54$ ) and 20 deaths (NAC: $n=2$; AC: $n=18$ ). DFS and OS did not differ significantly between the groups before IPTW correction (Figure A1E,F). 
Table 1. Patient characteristics, by chemotherapy strategy (NAC vs. AC).

\begin{tabular}{|c|c|c|c|c|}
\hline Variables & Category & $\begin{array}{c}\mathrm{AC} \\
(n=701)\end{array}$ & $\begin{array}{c}\text { NAC } \\
(n=202)\end{array}$ & $p$-Value \\
\hline Age & & $53.6(25.4-85.5)$ & $48.2(26.7-79.4)$ & $<0.0001$ \\
\hline BMI $\left(\mathrm{kg} / \mathrm{m}^{2}\right)$ & & $23.4((15.6-50)$ & $23.4(17.2-43)$ & 0.19 \\
\hline \multirow[t]{2}{*}{ Menopausal status } & Menopausal & $397(56.6)$ & $80(39.4)$ & $<0.0001$ \\
\hline & No menopausal & $304(43.4)$ & $122(60.6)$ & \\
\hline \multirow[t]{3}{*}{ Grade } & 1 & $14(2)$ & $3(1.5)$ & $<0.001$ \\
\hline & 2 & $231(33)$ & $1(0.5)$ & \\
\hline & 3 & $456(65)$ & $199(98$ & \\
\hline \multirow[t]{2}{*}{ HR } & Negative & $317(45.2)$ & $91(44.8)$ & 0.92 \\
\hline & Positive & $384(54.8)$ & $111(55.2)$ & \\
\hline \multirow[t]{4}{*}{ Nodal status } & NO & $573(81.7)$ & $81(39.9)$ & $<0.0001$ \\
\hline & N1 & $123(17.5)$ & $111(54.7)$ & \\
\hline & N2 & $3(0.4)$ & $7(3.4)$ & \\
\hline & N3 & $2(0.3)$ & $4(2)$ & \\
\hline Tumor size (mm) & & $20(3-100)$ & $40(10-120)$ & $<0.0001$ \\
\hline \multirow[t]{2}{*}{ T stage } & $\mathrm{T} 1$ & $453(64.6)$ & $24(11.8)$ & $<0.0001$ \\
\hline & $\mathrm{T} 2-\mathrm{T} 3$ & $248(35.4)$ & $179(88.2)$ & \\
\hline \multirow[t]{2}{*}{ Surgery } & Lumpectomy & $287(40.9)$ & $143(70.4)$ & $<0.0001$ \\
\hline & Mastectomy & $414(59.1)$ & $60(29.6)$ & \\
\hline \multirow[t]{2}{*}{ Axillary surgery } & Sentinel node biopsy & $182(26)$ & $9(4.4)$ & $<0.0001$ \\
\hline & Axillary dissection & $519(74)$ & $194(95.6)$ & \\
\hline Radiotherapy & & $689(98.3)$ & $202(99.5)$ & 0.32 \\
\hline \multirow[t]{4}{*}{ Chemotherapy } & Anthra regimen & $101(14)$ & $3(1)$ & $<0.001$ \\
\hline & Tax regimen & $12(2)$ & $16(8)$ & \\
\hline & Sequential Anthra-Tax regimen & $546(78)$ & $175(87)$ & \\
\hline & Other & $42(6)$ & $8(4)$ & \\
\hline Endocrine therapy & & $362(51.6)$ & $100(49.5)$ & 0.9 \\
\hline pCR & & - & $96(38.5)$ & \\
\hline \multirow[t]{2}{*}{ ypN status } & ypNo & - & $106(52.5)$ & \\
\hline & ypN positive & - & $96(47.5)$ & \\
\hline Median follow up (months) & & 48.7 & 39.2 & $<0.01$ \\
\hline \multirow[t]{3}{*}{ Events } & Local/loco-regional recurrence & $18(2.6)$ & $11(5.4)$ & \\
\hline & Distant metastasis & $40(5.7)$ & $14(6.9)$ & \\
\hline & Death & $18(2.6)$ & $2(1)$ & \\
\hline
\end{tabular}

Qualitative variables are presented as the median (range); quantitative variables are presented as $n$ (\%); AC: Adjuvant chemotherapy; NAC: neoadjuvant chemotherapy, BMI: body mass index; HR: hormone receptor; pCR: pathological complete response; anthra: anthracyclines, Tax: taxane.

\subsection{Survival Analysis after IPTW Correction}

The initial distribution of propensity scores was not well balanced between the NAC and AC groups (Figure A1C). Figure A1D in the Appendix A shows the standardized mean difference between the two groups for each variable before and after IPTW correction. After IPTW correction (represented by the red dots), all the variables were comparable between the NAC and AC groups, except for mastectomy rate and clinical tumor size, which remained higher in the NAC group.

\subsection{Disease-Free Survival}

After univariate IPTW-corrected analysis, the NAC and AC strategies were not significantly associated with DFS (cHR $=0.89,95 \% \mathrm{CI}$ : $(0.61-1.3), p=0.3$ ) (Figure 2A). When analyzing the association between CT strategy and DFS according to clinical and pathological factors, the NAC strategy was found to be significantly associated with DFS in the group of patients with baseline clinical nodal involvement $\left(\mathrm{cN}\right.$-positive) $\left(p_{\text {interaction }}=0.04\right)$ and in premenopausal patients $\left(p_{\text {interaction }}<0.01\right)$ (Figure $\left.3 \mathrm{~A}\right)$. 


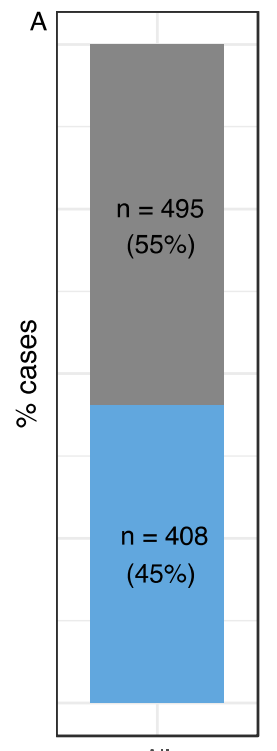

All

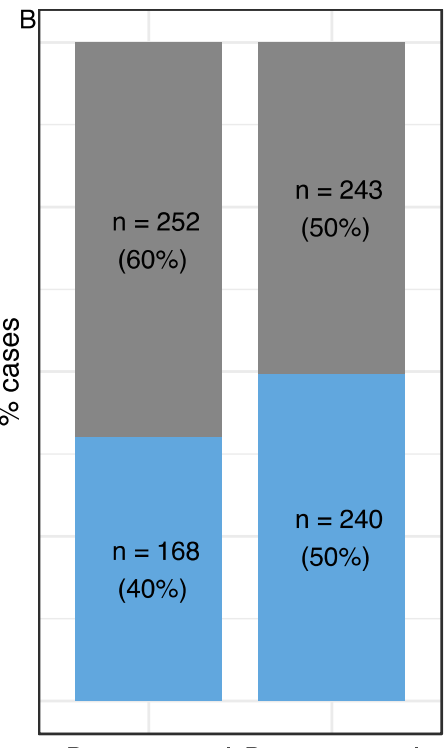

Premenopausal Postmenopausal

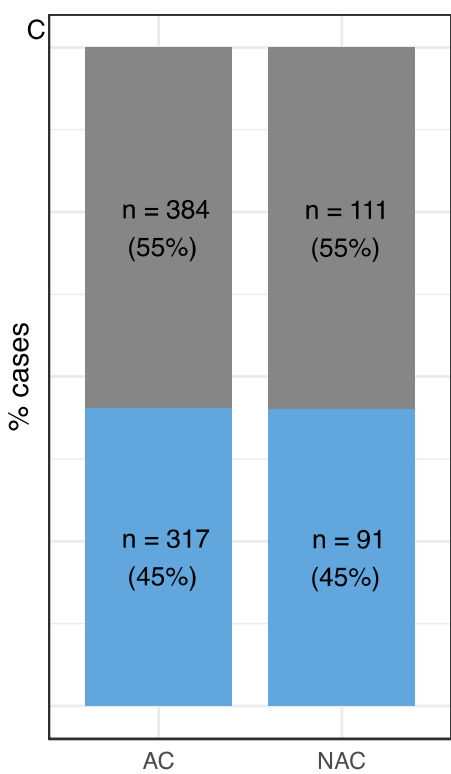

HR

Positive

Negative

Figure 1. Hormone receptor repartition. (A) Whole population, (B) according to menopausal status, (C) according to chemotherapy strategy. NAC: Neoadjuvant chemotherapy; AC: Adjuvant chemotherapy; HR: Hormone receptor.
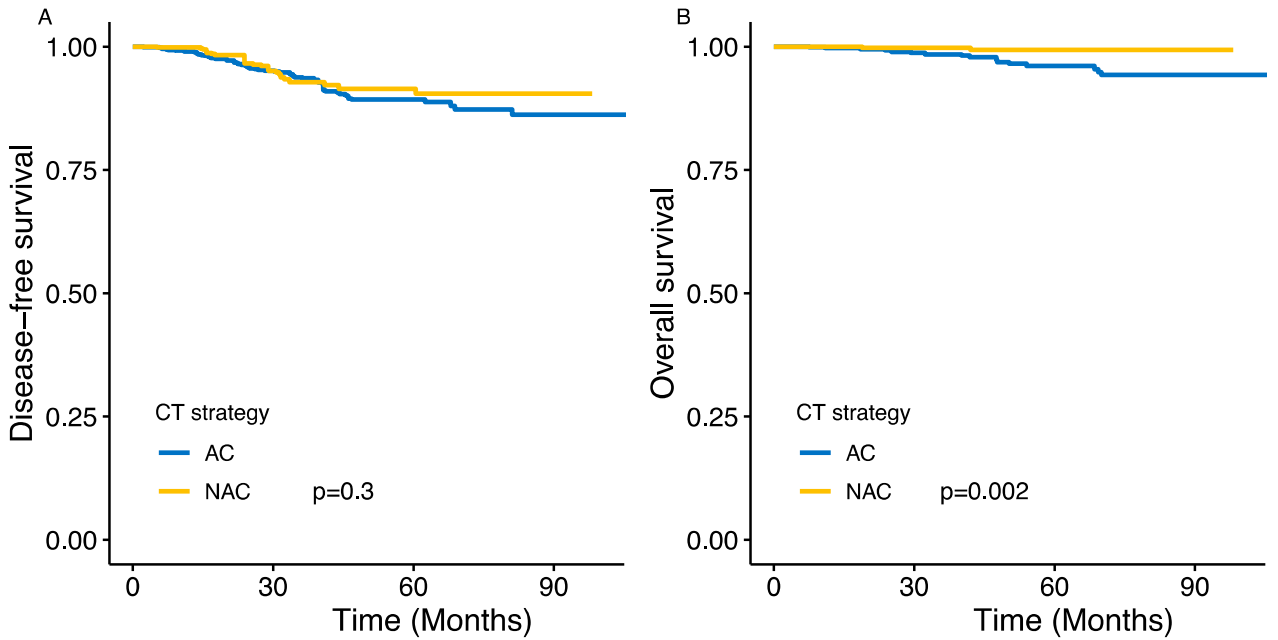

Figure 2. Association between treatment strategy (adjuvant or neoadjuvant) and survival after IPTW correction. (A) Disease-free survival; (B) Overall survival. CT: chemotherapy; NAC: Neoadjuvant chemotherapy; AC: Adjuvant chemotherapy; IPTW: inverse probability of treatment weights. The $p$-value is calculated with a weighted Cox model. No risk table is provided as the population is weighted).

After multivariate analysis and adjustment for surgical management, significant interactions remained between $\mathrm{cN}$ status and CT strategy, and between menopausal status and CT strategy ( $p_{\text {interaction }}=0.08$ and $<0.01$, respectively), indicating that the NAC strategy was more beneficial than the AC strategy in $\mathrm{cN}$-positive patients (cHR $=0.52,95 \% \mathrm{CI}$ $(0.3-0.9))$ and in postmenopausal women (cHR $=0.35,95 \% \mathrm{CI}(0.18-0.7)$ ) (Figure 3B). No other factor analyzed in this study was significantly associated with differences regarding the impact of the CT strategy.

Similar results were found regarding the DRFS: the NAC and AC strategies were not significantly associated with DRFS (Figure A2, while significant interactions existed between $\mathrm{cN}$ status and CT strategy, and between menopausal status and CT strategy (Figure A3A,B). 


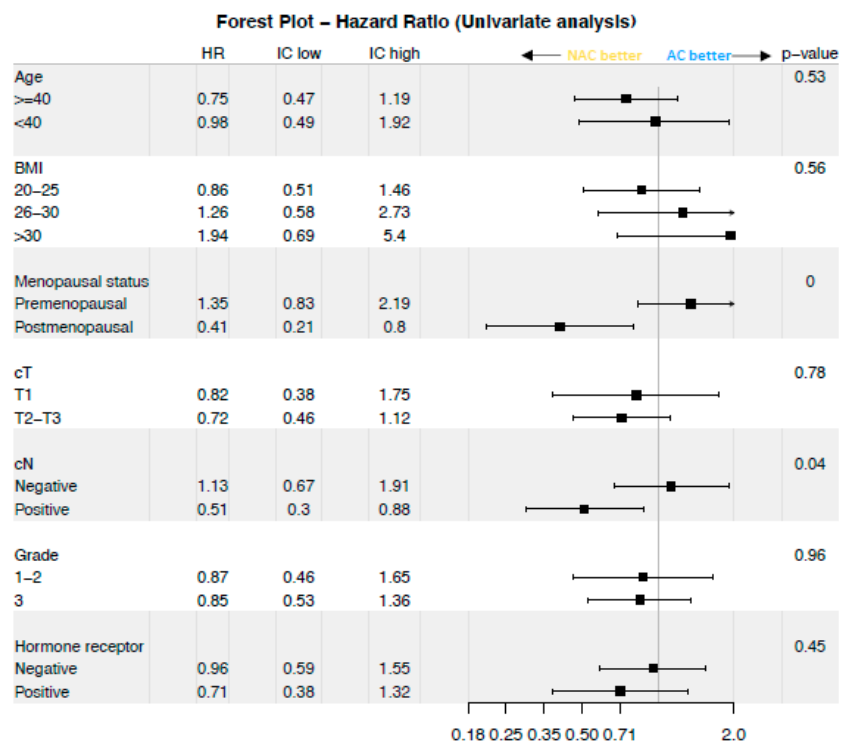

(A)

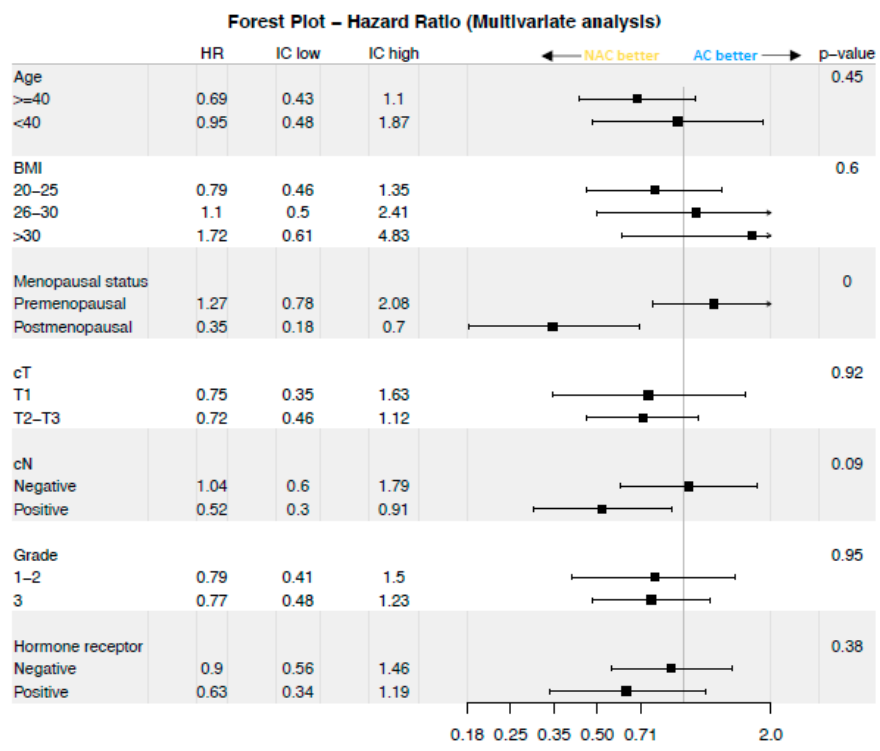

(B)

Figure 3. Impact of the strategy according to different clinical/histological variables. (A) Univariable analysis (B) Multivariable analysis. cN: initial clinical nodal status; cT: initial clinical tumor T stage; NAC: Neoadjuvant chemotherapy; AC: Adjuvant chemotherapy; BMI: body mass index; HR Hazard ratio; CI: confidence interval).

\subsection{Overall Survival}

Very few deaths $(n=2)$ were observed in the NAC group, limiting comparisons of the impact of the two strategies on overall survival. After IPTW-corrected univariate analysis, the NAC strategy was significantly associated with a greater benefit in terms of $\mathrm{OS}$ at five years than the AC strategy ( $\mathrm{cHR}=0.12,95 \% \mathrm{CI}(0.03-0.47), p=0.002)$ (Figure 2B). After multivariate analysis and adjustment for type of surgery and radiotherapy, this survival benefit remained statistically significant ( $\mathrm{cHR}=0.09,95 \% \mathrm{CI}(0.02-0.35), p<0.001)$ (the figure is not shown because the confidence interval was infinite, given the small number of deaths).

\section{Discussion}

In this study, we compared the NAC and AC strategies in HER2-positive BC patients. We found that the NAC strategy was significantly associated with longer DFS in patients with a clinical positive nodal status at baseline, and in postmenopausal patients. Moreover, NAC was associated with a significant survival benefit over AC in the whole population.

This is, to our knowledge, one of the first studies to report an impact of treatment strategy (NAC vs. AC) in a subset of patients: the HER2-positive population. In a recent study of Pomponio et al., using IPTW to deal with the selection bias showed no difference between AC and NAC strategy in early HER2-postive BC [30]. Many studies have reported higher rates of breast-conserving surgery in patients treated with NAC, but a recent EBCTCG meta-analysis [13] found that NAC was as effective as AC in reducing the risk of distant recurrence and death from $B C$. NAC was associated with a higher local recurrence rate at 15 years than $\mathrm{AC}$ ( $21.4 \%$ vs. $15.9 \%$ respectively), but these results must be interpreted with care, as chemotherapy regimens, surgical, and radiation techniques have improved over time. Stankowski-Drengler et al. [31] identified three studies analyzing outcomes, by chemotherapy strategy, and based on receptor subtype. One of these studies reported analyses for the HER2positive subgroup showing an OS benefit of the NAC strategy ( $\mathrm{HR}=0.1,95 \% \mathrm{CI}[0.02-0.58]$, $p=0.01$ ). However, the HER2-positive subgroup only contained 30 patients, and patients treated with targeted therapies against HER2 were excluded [32].

Little scientific evidence is available concerning the subgroups of patients with HER2positive BC that should be offered a NAC strategy rather than an AC strategy. A large 
tumor size is considered to be a genuine indication for NAC, as it increases the likelihood of breast-conserving surgery, and the neoadjuvant strategy was validated as a standard of care in the 2019 St Gallen Consensus for stage II-III tumors [33]. The availability of drugs also guides therapeutic strategies. The 2019 National Comprehensive Cancer Network guidelines validated a NAC regimen combining trastuzumab and pertuzumab for HER2positive tumors with a diameter exceeding $2 \mathrm{~cm}$ and for node-positive patients [34]. The use of adjuvant drugs, such as TDM-1, in the post-neoadjuvant setting also provides a major argument in favor of NAC. The KATHERINE trial found a benefit of the association of trastuzumab and emtansine (TDM-1) in the adjuvant setting for patients with residual disease after NAC, with a risk of recurrence of invasive BC or death $50 \%$ lower in the TDM-1 group $(\mathrm{HR}=0.50 ; 95 \% \mathrm{CI}(0.39-0.64))$ [9]. Since then, neoadjuvant strategy tends to be the standard for the HER2-positive tumors in most recommendations [6,35].

In our cohort, menopausal status was significantly associated with DFS. These finding are consistent with the retrospective study by Kim et al. on 229 HER2-positive tumors treated by NAC plus trastuzumab, which reported significantly lower pCR rates in young and premenopausal patients [36].

In contrast, another study with 475 HER2-positive tumors found no difference in pCR rates between two age groups ( $<40$ years vs. $\geq 40$ years) [37], but none of these patients were treated with neoadjuvant trastuzumab. In 2015, a meta-analysis from the German Breast Group (GBG) and the German Gynecological Oncology Working Group-Breast (AGO-B) study group focusing on young patients found no significant difference in pCR rates as a function of age or HR status, in patients with HER2-positive tumors [38].

We also found that the NAC strategy was beneficial in the population of patients with positive nodal status at baseline. Axillary node involvement is associated with more advanced tumor stages at BC diagnosis, and is correlated with poorer BC prognosis and higher rates of distant metastasis [39-41]. Several hypotheses can be put forward to explain our findings. First, the NAC strategy results in a shorter time-to-first systemic treatment. As HER2-positive BCs are highly sensitive to chemotherapy combined with anti-HER2 treatment, the impact of a delay in initiating trastuzumab treatment and/or chemotherapy might be particularly important in this subpopulation. However, the REMAGUS02 trial randomly assigned HER2-positive BC patients to two arms (NAC alone or NAC plus trastuzumab), and reported that there was no survival advantage associated with the early introduction of trastuzumab [42]. Second, there is evidence to suggest that trastuzumab has an immunological mode of action, including the induction of antibody-dependent cellular cytotoxicity, endogenous humoral, and enhanced T cell-mediated immune responses, and higher levels of tumor infiltration with immune effectors, including natural killer cells. It remains unknown whether these mechanisms act differently before or after the surgical removal of the tumor, but it could be argued that such mechanisms could be attenuated with the tumor in place $[43,44]$. Third, although purely speculative, we can hypothesize that axillary involvement is of importance in the context of this cancer with a large immunological dimension for which antibody-based therapies are available [45].

Our study has several limitations. First, as this study was retrospective, patients were not randomly allocated to the NAC and AC strategies. We tried to limit selection bias by weighting according to the probability of treatment allocation with a propensity score. This method has been shown to be an effective alternative to consistently reduced systematic baseline differences [20]. Second, none of the patients received pertuzumab in the neoadjuvant setting. The addition of pertuzumab (a humanized monoclonal antibody targeting an epitope of HER2 different from the one targeted by trastuzumab) to trastuzumab associated with docetaxel has been shown to yield higher rates of $\mathrm{pCR}$, and a higher five-year DFS, in early, locally advanced and inflammatory BC [46,47]. In 2019, Tolaney et al. published an updated seven-year follow-up for an adjuvant paclitaxel and trastuzumab protocol, demonstrating the efficacy of an adjuvant anthracycline-free regimen for HER2-positive, node-negative tumors of $3 \mathrm{~cm}$ or less in diameter [48]. With its very low rates of recurrence and highly favorable toxicity profile, this adjuvant strategy has become a standard of care 
in the management of small node-negative HER2-positive BC. It remains unknown whether these results would be reproducible with such a chemotherapy regimen. Finally, we found discrepancies between the results for DFS and OS. These differences could be explained by the higher death rate in the AC group than in the NAC group, and a higher local recurrence rate in the NAC group, possibly due to the higher rate of conservative surgery. However, similar results were found regarding DRFS.

Our study also has several strengths. This is to our knowledge the first study demonstrating an OS benefit of trastuzumab containing neoadjuvant chemotherapy for HER2positive BC patients, with a large statistical power and a long-term follow-up. Moreover, as NAC has progressively become a standard of care over the last years, historical data comparing AC and NAC in these tumors may be difficult to encounter from now on, making these data unique. Finally, beyond new drugs and new predictive markers, this is one of the first studies supporting the hypothesis that therapeutic strategies tailored to each individual may influence the natural history of BC.

\section{Conclusions}

In conclusion, NAC remains the standard of care for HER2-positive BCs [49], and our study provides additional evidence to support the treatment of patients with HER2-positive tumors and baseline clinical nodal involvement with NAC rather than AC. Validation studies determining whether similar subgroups of patients derive benefits from the combination of a NAC regimen with anti-HER2 dual-blockade with trastuzumab and pertuzumab are eagerly awaited.

Author Contributions: Conceptualization, E.L., A.B., F.R. and A.-S.H.; Methodology, E.L., A.B., F.R. and A.-S.H.; Software, E.L. and A.-S.H.; validation, E.L., A.B., J.-G.F., C.L.G., L.D., F.C., B.G., L.L., J.-Y.P., F.R. and A.-S.H.; formal analysis, E.L.; investigation, E.L., A.B.; resources, E.L., J.-G.F., F.R. and A.-S.H.; data curation, E.L., F.R. and A.-S.H.; writing-original draft preparation, E.L., A.B., F.R. and A.-S.H.; writing—review and editing, E.L., A.B., J.-G.F., C.L.G., L.D., F.C., B.G., L.L., J.-Y.P., F.R. and A.-S.H.; visualization, E.L., F.R. and A.-S.H.; supervision, F.R. and A.-S.H.; project administration, A.-S.H. and J.-G.F.; funding acquisition, F.R. and A.-S.H. All authors have read and agreed to the published version of the manuscript.

Funding: We thank Roche France for financial support for the construction of the Institut Curie neoadjuvant database (NEOREP). B.G. was supported by Alfonso Martin Escudero Foundation research grant. The funders had no role in study design, data collection and analysis, decision to publish, or preparation of the manuscript.

Institutional Review Board Statement: Ethic approval number here: CNIL declaration number 1547270; Approved by the Breast Cancer Study Group of Institute Curie, the study was conducted according to institutional and ethical rules concerning research on tissue specimens and patients. Informed consent from patients was not required.

Informed Consent Statement: Patient consent was not required. All experiments were performed retrospectively and in accordance with the French Bioethics Law 2004-800, the French National Institute of Cancer (INCa) Ethics Charter and after approval by the Institut Curie review board and ethics committee (Comité de Pilotage of the Groupe Sein). In the French legal context, our institutional review board waived the need for written informed consent from the participants. Moreover, women were informed of the research use of their tissues and did not declare any opposition for such researches. Data were analyzed anonymously.

Data Availability Statement: Data available on request due to privacy/ethical restrictions. The data that support the findings of this study are available on request from the corresponding author, [FR]. The data are not publicly available because they contain information that could compromise the privacy of research participants.

Conflicts of Interest: The authors declare no conflict of interest. 


\section{Appendix A}
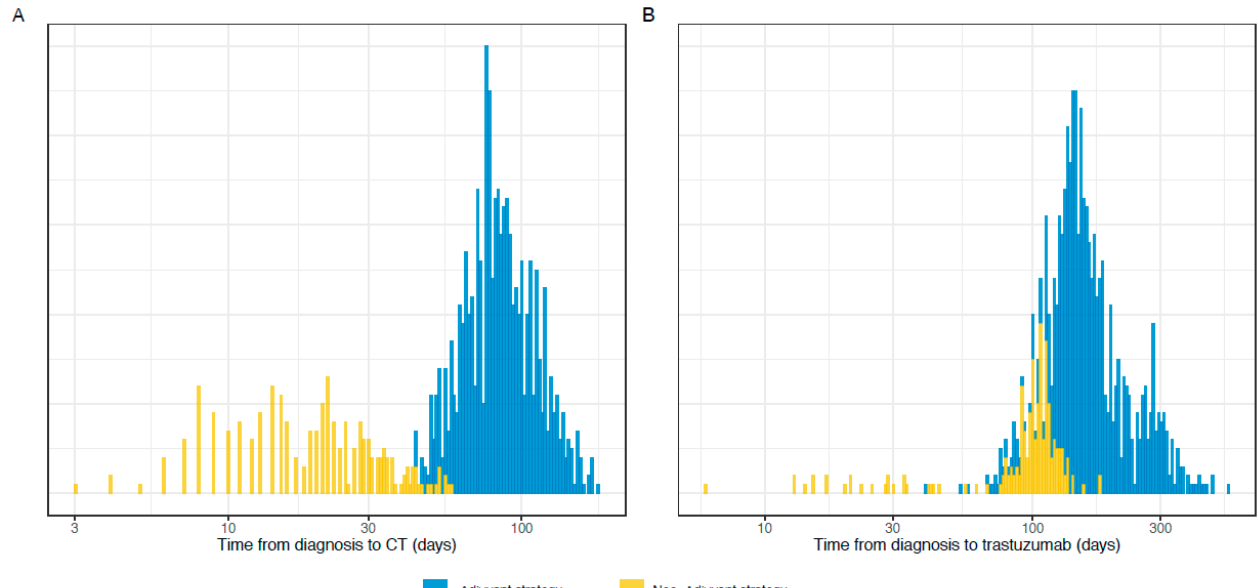
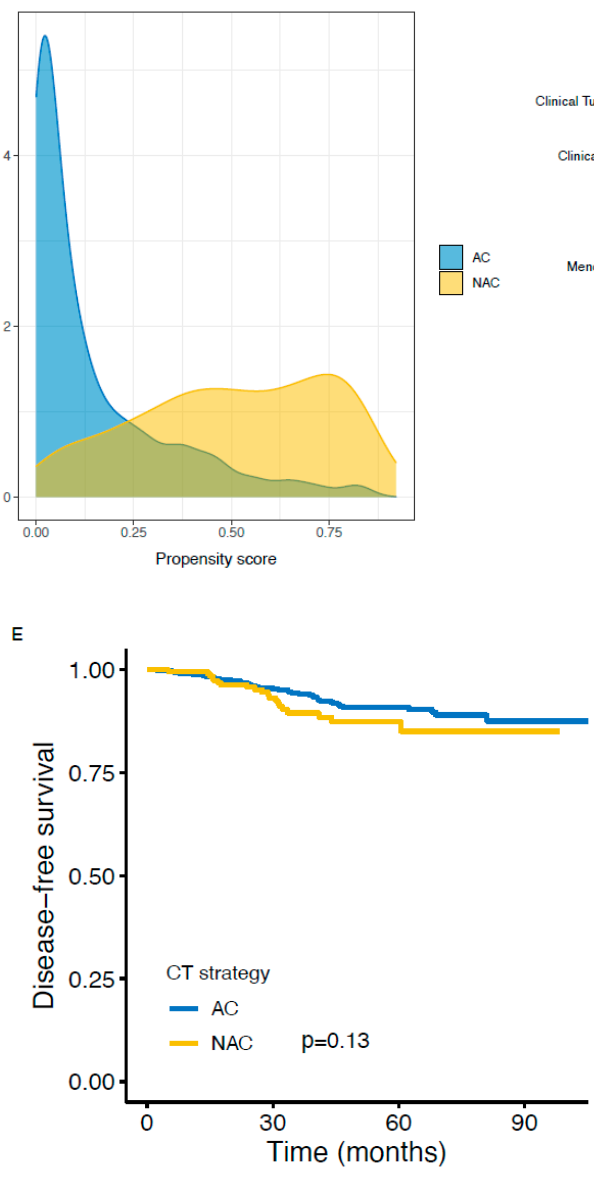

$\mathrm{N}$ at risk

AC 701

NAC 202

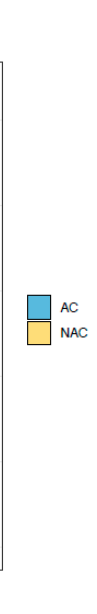

D

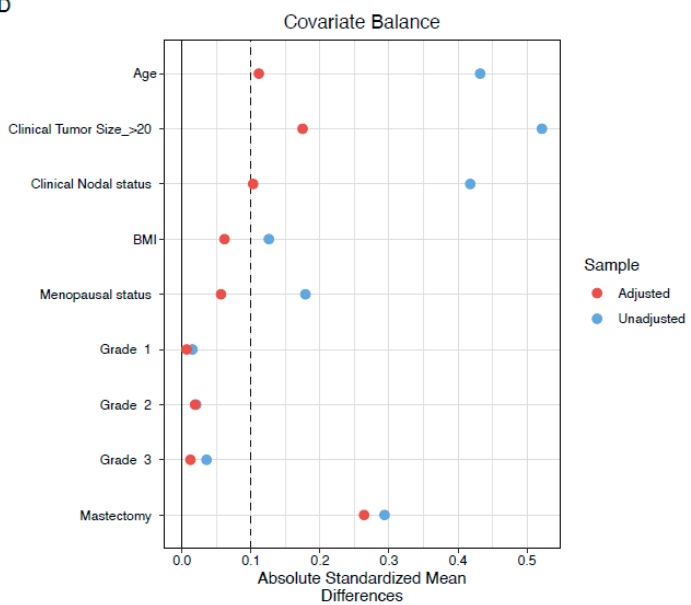

F

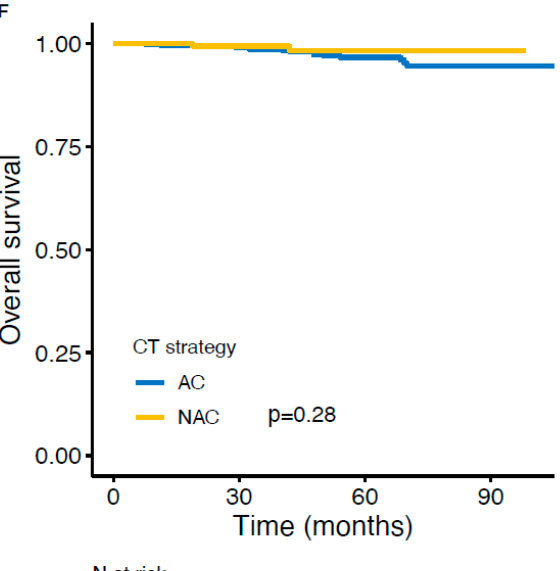

$\mathrm{N}$ at risk

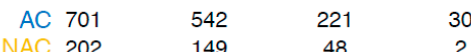

Figure A1. (A) Time between diagnosis and first chemotherapy, between neoadjuvant and adjuvant strategy. (B) Time between diagnosis and first trastuzumab, between neoadjuvant and adjuvant strategy. (C) Distribution of the propensity score in the population, according to the chemotherapy strategy. (D) Covariate balance before and after IPTW adjustment. (E) Association between treatment strategy (adjuvant or neoadjuvant) and disease-free survival, before IPTW adjustment. (F) Association between treatment strategy (adjuvant or neoadjuvant) and overall survival, before IPTW adjustment. 


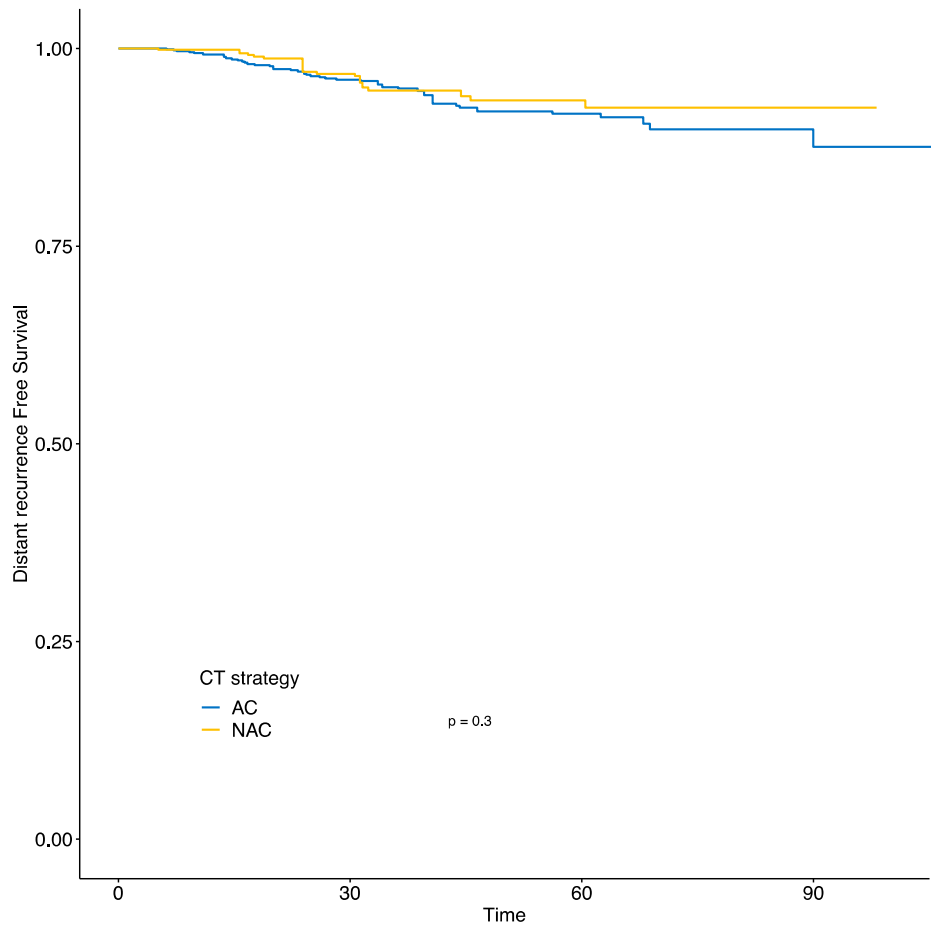

Figure A2. Association between treatment strategy (adjuvant or neoadjuvant) and survival after IPTW correction, for distant recurrence-free survival.
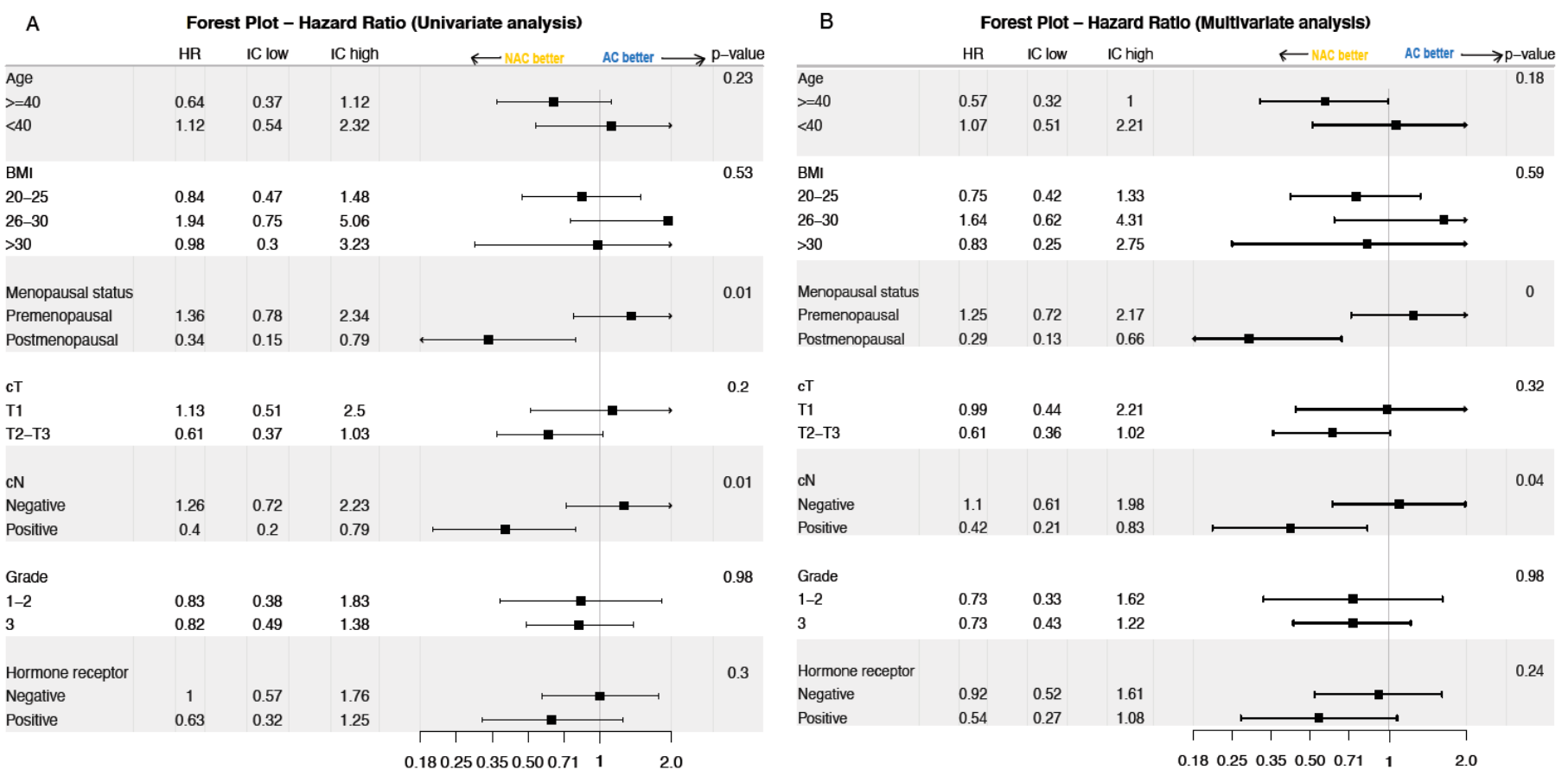

Figure A3. Impact of the strategy according to different clinical/histological variables for distant recurrence-free survival. (A) Univariable analysis. (B) Multivariable analysis. cN: initial clinical nodal status; $\mathrm{cT}$ : initial clinical tumor T stage; NAC: Neoadjuvant chemotherapy; AC: Adjuvant chemotherapy; BMI: body mass index; HR Hazard ratio; CI: confidence interval). 


\section{References}

1. $\quad$ Ross, J.S.; Slodkowska, E.A.; Symmans, W.F.; Pusztai, L.; Ravdin, P.M.; Hortobagyi, G.N. The HER-2 Receptor and Breast Cancer: Ten Years of Targeted Anti-HER-2 Therapy and Personalized Medicine. Oncologist 2009, 14, 320-368. [CrossRef]

2. Fokter Dovnik, N.; Dovnik, A.; Čas Sikošek, N.; Ravnik, M.; Arko, D.; Takač, I. Prognostic Role of HER2 Status and Adjuvant Trastuzumab Treatment in Lymph Node-Negative Breast Cancer Patients-a Retrospective Single Center Analysis. Breast Care 2016, 11, 406-410. [CrossRef]

3. Kaufmann, M.; von Minckwitz, G.; Bear, H.D.; Buzdar, A.; McGale, P.; Bonnefoi, H.; Colleoni, M.; Denkert, C.; Eiermann, W.; Jackesz, R.; et al. Recommendations from an International Expert Panel on the Use of Neoadjuvant (Primary) Systemic Treatment of Operable Breast Cancer: New Perspectives 2006. Ann. Oncol. 2007, 18, 1927-1934. [CrossRef] [PubMed]

4. Truin, W.; Vugts, G.; Roumen, R.M.H.; Maaskant-Braat, A.J.G.; Nieuwenhuijzen, G.A.P.; van der Heiden-van der Loo, M.; Tjan-Heijnen, V.C.G.; Voogd, A.C. Differences in Response and Surgical Management with Neoadjuvant Chemotherapy in Invasive Lobular Versus Ductal Breast Cancer. Ann. Surg. Oncol. 2016, 23, 51-57. [CrossRef] [PubMed]

5. Cristofanilli, M.; Gonzalez-Angulo, A.; Sneige, N.; Kau, S.-W.; Broglio, K.; Theriault, R.L.; Valero, V.; Buzdar, A.U.; Kuerer, H.; Buchholz, T.A.; et al. Invasive Lobular Carcinoma Classic Type: Response to Primary Chemotherapy and Survival Outcomes. J. Clin. Oncol. 2005, 23, 41-48. [CrossRef] [PubMed]

6. Telli, M.L.; Gradishar, W.J.; Ward, J.H. NCCN Guidelines Updates: Breast Cancer. J. Natl. Compr. Canc. Netw. 2019, 17, 552-555. [CrossRef] [PubMed]

7. Tubiana-Hulin, M.; Stevens, D.; Lasry, S.; Guinebretière, J.M.; Bouita, L.; Cohen-Solal, C.; Cherel, P.; Rouëssé, J. Response to Neoadjuvant Chemotherapy in Lobular and Ductal Breast Carcinomas: A Retrospective Study on 860 Patients from One Institution. Ann. Oncol. 2006, 17, 1228-1233. [CrossRef]

8. Masuda, N.; Lee, S.-J.; Ohtani, S.; Im, Y.-H.; Lee, E.-S.; Yokota, I.; Kuroi, K.; Im, S.-A.; Park, B.-W.; Kim, S.-B.; et al. Adjuvant Capecitabine for Breast Cancer after Preoperative Chemotherapy. N. Engl. J. Med. 2017, 376, 2147-2159. [CrossRef]

9. von Minckwitz, G.; Huang, C.-S.; Mano, M.S.; Loibl, S.; Mamounas, E.P.; Untch, M.; Wolmark, N.; Rastogi, P.; Schneeweiss, A.; Redondo, A.; et al. Trastuzumab Emtansine for Residual Invasive HER2-Positive Breast Cancer. N. Engl. J. Med. 2018, 380, 617-628. [CrossRef]

10. Mamounas, E.P. NSABP Protocol B-27. Preoperative Doxorubicin plus Cyclophosphamide Followed by Preoperative or Postoperative Docetaxel. Oncol. (Williston Park NY) 1997, 11, 37-40.

11. Mauri, D.; Pavlidis, N.; Ioannidis, J.P.A. Neoadjuvant versus Adjuvant Systemic Treatment in Breast Cancer: A Meta-Analysis. J. Natl. Cancer Inst. 2005, 97, 188-194. [CrossRef] [PubMed]

12. Fisher, B.; Brown, A.; Mamounas, E.; Wieand, S.; Robidoux, A.; Margolese, R.G.; Cruz, A.B.; Fisher, E.R.; Wickerham, D.L.; Wolmark, N.; et al. Effect of Preoperative Chemotherapy on Local-Regional Disease in Women with Operable Breast Cancer: Findings from National Surgical Adjuvant Breast and Bowel Project B-18. J. Clin. Oncol. 1997, 15, 2483-2493. [CrossRef] [PubMed]

13. Early Breast Cancer Trialists' Collaborative Group (EBCTCG). Long-Term Outcomes for Neoadjuvant versus Adjuvant Chemotherapy in Early Breast Cancer: Meta-Analysis of Individual Patient Data from Ten Randomised Trials. Lancet Oncol. 2018, 19, 27-39. [CrossRef]

14. Elston, C.W.; Ellis, I.O. Pathological Prognostic Factors in Breast Cancer. I. The Value of Histological Grade in Breast Cancer: Experience from a Large Study with Long-Term Follow-Up. Histopathology 2002, 41, 154-161. [CrossRef] [PubMed]

15. Gligorov, J. RPC Saint-Paul-de-Vence: Deuxième. Oncologie 2007, 9, 591-592. [CrossRef]

16. Wolff, A.C.; Hammond, M.E.H.; Hicks, D.G.; Dowsett, M.; McShane, L.M.; Allison, K.H.; Allred, D.C.; Bartlett, J.M.S.; Bilous, M.; Fitzgibbons, P.; et al. Recommendations for Human Epidermal Growth Factor Receptor 2 Testing in Breast Cancer: American Society of Clinical Oncology/College of American Pathologists Clinical Practice Guideline Update. Arch. Pathol. Lab. Med. 2014, 138, 241-256. [CrossRef]

17. Classe, J.-M.; Barranger, E.; Houvenaeghel, G. L'exploration et Le Traitement de La Région Axillaire Des Tumeurs Infiltrantes Du Sein. Technique Du Ganglion Sentinelle et Chimiothérapie Néoadjuvante.Recommandations Franco-Phones Pour La Pratique Clinique Des Cancers Du Sein 2013. Oncologie 2013, 15, 586-588. [CrossRef]

18. Goldhirsch, A.; Ingle, J.N.; Gelber, R.D.; Coates, A.S.; Thürlimann, B.; Senn, H.-J. Thresholds for Therapies: Highlights of the St Gallen International Expert Consensus on the Primary Therapy of Early Breast Cancer 2009. Ann. Oncol. 2009, 20, 1319-1329. [CrossRef]

19. Joffe, M.M.; Rosenbaum, P.R. Invited Commentary: Propensity Scores. Am. J. Epidemiol. 1999, 150, 327-333. [CrossRef]

20. Austin, P.C.; Grootendorst, P.; Anderson, G.M. A Comparison of the Ability of Different Propensity Score Models to Balance Measured Variables between Treated and Untreated Subjects: A Monte Carlo Study. Stat. Med. 2007, 26, 734-753. [CrossRef]

21. Brookhart, M.A.; Schneeweiss, S.; Rothman, K.J.; Glynn, R.J.; Avorn, J.; Stürmer, T. Variable Selection for Propensity Score Models. Am. J. Epidemiol. 2006, 163, 1149-1156. [CrossRef] [PubMed]

22. Perkins, S.M.; Tu, W.; Underhill, M.G.; Zhou, X.H.; Murray, M.D. The Use of Propensity Scores in Pharmacoepidemiologic Research. Pharmacoepidemiol. Drug Saf. 2000, 9, 93-101. [CrossRef]

23. Austin, P.C.; Stuart, E.A. Moving towards Best Practice When Using Inverse Probability of Treatment Weighting (IPTW) Using the Propensity Score to Estimate Causal Treatment Effects in Observational Studies. Stat. Med. 2015, 34, 3661-3679. [CrossRef] [PubMed] 
24. Austin, P.C.; Stuart, E.A. The Performance of Inverse Probability of Treatment Weighting and Full Matching on the Propensity Score in the Presence of Model Misspecification When Estimating the Effect of Treatment on Survival Outcomes. Stat. Methods Med. Res. 2017, 26, 1654-1670. [CrossRef]

25. Almahariq, M.F.; Quinn, T.J.; Siddiqui, Z.; Jawad, M.S.; Chen, P.Y.; Gustafson, G.S.; Dilworth, J.T. Breast Conserving Therapy Is Associated with Improved Overall Survival Compared to Mastectomy in Early-Stage, Lymph Node-Negative Breast Cancer. Radiother. Oncol. J. Eur. Soc. Ther. Radiol. Oncol. 2020, 142, 186-194. [CrossRef]

26. Huang Bartlett, C.; Mardekian, J.; Cotter, M.J.; Huang, X.; Zhang, Z.; Parrinello, C.M.; Bourla, A.B. Concordance of Real-World versus Conventional Progression-Free Survival from a Phase 3 Trial of Endocrine Therapy as First-Line Treatment for Metastatic Breast Cancer. PLoS ONE 2020, 15, e0227256. [CrossRef]

27. Cole, S.R.; Hernán, M.A. Adjusted Survival Curves with Inverse Probability Weights. Comput. Methods Programs Biomed. 2004, 75, 45-49. [CrossRef]

28. Selvin, S. Statistical Analysis of Epidemiologic Data; Oxford University Press: New York, NY, USA, 1996; pp. $213-214$.

29. R Core Team. R: A Language and Environment for Statistical Computing. R Foundation for Statistical Computing; R Core Team: Vienna, Austria, 2008; Available online: Https:/ /Www.R-Project.Org/ (accessed on 10 January 2021).

30. Pomponio, M.K.; Burkbauer, L.; Goldbach, M.; Nazarian, S.M.; Xie, F.; Clark, A.S.; Matro, J.M.; Fox, K.R.; Shulman, L.N.; Keele, L.J.; et al. Refining the Indications for Neoadjuvant Chemotherapy for Patients with HER2+ Breast Cancer: A Single Institution Experience. J. Surg. Oncol. 2020, 121, 447-455. [CrossRef]

31. Stankowski-Drengler, T.J.; Livingston-Rosanoff, D.; Schumacher, J.R.; Hanlon, B.M.; Hitchcock, M.E.; Neuman, H.B. Breast Cancer Outcomes of Neoadjuvant Versus Adjuvant Chemotherapy by Receptor Subtype: A Scoping Review. J. Surg. Res. 2020, 254, 83-90. [CrossRef]

32. Yang, H.; Zhou, L.; Wang, S.; Cao, Y.; Tong, F.; Liu, P.; Zhou, B.; Cheng, L.; Liu, M.; Liu, H.; et al. Retrospective Analysis of Concurrent Docetaxel and Epirubicin Neoadjuvant versus Adjuvant Chemotherapy: Which Leads to Better Outcomes for Different Subtype Breast Cancer Patients? Medicine 2018, 97, e12690. [CrossRef]

33. Gnant, M.; Harbeck, N.; Thomssen, C. St. Gallen/Vienna 2017: A Brief Summary of the Consensus Discussion about Escalation and De-Escalation of Primary Breast Cancer Treatment. Breast Care Basel Switz. 2017, 12, 102-107. [CrossRef] [PubMed]

34. Gradishar, W.J.; Anderson, B.O.; Balassanian, R.; Blair, S.L.; Burstein, H.J.; Cyr, A.; Elias, A.D.; Farrar, W.B.; Forero, A.; Giordano, S.H.; et al. NCCN Guidelines Insights: Breast Cancer, Version 1.2017. JNCCN 2017, 15, 433-451. [CrossRef]

35. Cardoso, F.; Kyriakides, S.; Ohno, S.; Penault-Llorca, F.; Poortmans, P.; Rubio, I.T.; Zackrisson, S.; Senkus, E. Early Breast Cancer: ESMO Clinical Practice Guidelines for Diagnosis, Treatment and Follow-Upt. Ann. Oncol. 2019, 30, 1194-1220. [CrossRef] [PubMed]

36. Kim, M.M.; Allen, P.; Gonzalez-Angulo, A.M.; Woodward, W.A.; Meric-Bernstam, F.; Buzdar, A.U.; Hunt, K.K.; Kuerer, H.M.; Litton, J.K.; Hortobagyi, G.N.; et al. Pathologic Complete Response to Neoadjuvant Chemotherapy with Trastuzumab Predicts for Improved Survival in Women with HER2-Overexpressing Breast Cancer. Ann. Oncol. 2013, 24, 1999-2004. [CrossRef]

37. Huober, J.; von Minckwitz, G.; Denkert, C.; Tesch, H.; Weiss, E.; Zahm, D.M.; Belau, A.; Khandan, F.; Hauschild, M.; Thomssen, C.; et al. Effect of Neoadjuvant Anthracycline-Taxane-Based Chemotherapy in Different Biological Breast Cancer Phenotypes: Overall Results from the GeparTrio Study. Breast Cancer Res. Treat. 2010, 124, 133-140. [CrossRef]

38. Loibl, S.; Jackisch, C.; Lederer, B.; Untch, M.; Paepke, S.; Kümmel, S.; Schneeweiss, A.; Huober, J.; Hilfrich, J.; Hanusch, C.; et al. Outcome after Neoadjuvant Chemotherapy in Young Breast Cancer Patients: A Pooled Analysis of Individual Patient Data from Eight Prospectively Randomized Controlled Trials. Breast Cancer Res. Treat. 2015, 152, 377-387. [CrossRef]

39. Silvestrini, R.; Daidone, M.G.; Luisi, A.; Boracchi, P.; Mezzetti, M.; Di Fronzo, G.; Andreola, S.; Salvadori, B.; Veronesi, U. Biologic and Clinicopathologic Factors as Indicators of Specific Relapse Types in Node-Negative Breast Cancer. J. Clin. Oncol. 1995, 13, 697-704. [CrossRef]

40. Fisher, B.; Bauer, M.; Wickerham, D.L.; Redmond, C.K.; Fisher, E.R.; Cruz, A.B.; Foster, R.; Gardner, B.; Lerner, H.; Margolese, R. Relation of Number of Positive Axillary Nodes to the Prognosis of Patients with Primary Breast Cancer. An NSABP Update. Cancer 1983, 52, 1551-1557. [CrossRef]

41. Todd, J.H.; Dowle, C.; Williams, M.R.; Elston, C.W.; Ellis, I.O.; Hinton, C.P.; Blamey, R.W.; Haybittle, J.L. Confirmation of a Prognostic Index in Primary Breast Cancer. Br. J. Cancer 1987, 56, 489-492. [CrossRef]

42. Giacchetti, S.; Hamy, A.-S.; Delaloge, S.; Brain, E.; Berger, F.; Sigal-Zafrani, B.; Mathieu, M.-C.; Bertheau, P.; Guinebretière, J.M.; Saghatchian, M.; et al. Long-Term Outcome of the REMAGUS 02 Trial, a Multicenter Randomised Phase II Trial in Locally Advanced Breast Cancer Patients Treated with Neoadjuvant Chemotherapy with or without Celecoxib or Trastuzumab According to HER2 Status. Eur. J. Cancer Oxf. Engl. 1990 2017, 75, 323-332. [CrossRef]

43. Arnould, L.; Gelly, M.; Penault-Llorca, F.; Benoit, L.; Bonnetain, F.; Migeon, C.; Cabaret, V.; Fermeaux, V.; Bertheau, P.; Garnier, J.; et al. Trastuzumab-Based Treatment of HER2-Positive Breast Cancer: An Antibody-Dependent Cellular Cytotoxicity Mechanism? Br. J. Cancer 2006, 94, 259-267. [CrossRef] [PubMed]

44. Gennari, R.; Menard, S.; Fagnoni, F.; Ponchio, L.; Scelsi, M.; Tagliabue, E.; Castiglioni, F.; Villani, L.; Magalotti, C.; Gibelli, N.; et al. Pilot Study of the Mechanism of Action of Preoperative Trastuzumab in Patients with Primary Operable Breast Tumors Overexpressing HER2. Clin. Cancer Res. 2004, 10, 5650-5655. [CrossRef] [PubMed]

45. Moasser, M.M. Two Dimensions in Targeting HER2. J. Clin. Oncol. 2014, 32, 2074-2077. [CrossRef] 
46. Gianni, L.; Pienkowski, T.; Im, Y.-H.; Roman, L.; Tseng, L.-M.; Liu, M.-C.; Lluch, A.; Staroslawska, E.; de la Haba-Rodriguez, J.; Im, S.-A.; et al. Efficacy and Safety of Neoadjuvant Pertuzumab and Trastuzumab in Women with Locally Advanced, Inflammatory, or Early HER2-Positive Breast Cancer (NeoSphere): A Randomised Multicentre, Open-Label, Phase 2 Trial. Lancet Oncol. 2012, 13, 25-32. [CrossRef]

47. Gianni, L.; Pienkowski, T.; Im, Y.-H.; Tseng, L.-M.; Liu, M.-C.; Lluch, A.; Starosławska, E.; de la Haba-Rodriguez, J.; Im, S.-A.; Pedrini, J.L.; et al. 5-Year Analysis of Neoadjuvant Pertuzumab and Trastuzumab in Patients with Locally Advanced, Inflammatory, or Early-Stage HER2-Positive Breast Cancer (NeoSphere): A Multicentre, Open-Label, Phase 2 Randomised Trial. Lancet Oncol. 2016, 17, 791-800. [CrossRef]

48. Tolaney, S.M.; Guo, H.; Pernas, S.; Barry, W.T.; Dillon, D.A.; Ritterhouse, L.; Schneider, B.P.; Shen, F.; Fuhrman, K.; Baltay, M.; et al. Seven-Year Follow-Up Analysis of Adjuvant Paclitaxel and Trastuzumab Trial for Node-Negative, Human Epidermal Growth Factor Receptor 2-Positive Breast Cancer. J. Clin. Oncol. 2019, 37, 1868-1875. [CrossRef]

49. Brandão, M.; Reyal, F.; Hamy, A.-S.; Piccart-Gebhart, M. Neoadjuvant Treatment for Intermediate/High-Risk HER2-Positive and Triple-Negative Breast Cancers: No Longer an "option" but an Ethical Obligation. ESMO Open 2019, 4, e000515. [CrossRef] 